\title{
An update on long-acting therapies in chronic sight- threatening eye diseases of the posterior segment: AMD, DMO, RVO, uveitis and glaucoma
}

Faruque Ghanchi ${ }^{1 \otimes}{ }^{凶}$, Rupert Bourne ${ }^{2}$, Susan M. Downes ${ }^{3}$, Richard Gale ${ }^{4}$, Christina Rennie (iD ${ }^{5}$, lan Tapply ${ }^{2}$ and Sobha Sivaprasad ${ }^{6}$

(c) The Author(s) 2021

In the real-world setting, there is suboptimal compliance with treatments that require frequent administration and assessment visits. This undertreatment frequently has negative consequences in eye disease and carries a real risk to vision. For example, patients with glaucoma risk progression of visual loss even with a small number of missed doses, and patients with neovascular age-related degeneration (nAMD) who fail to attend a bi-monthly clinic appointment to receive an intravitreal anti-vascular endothelial growth factor (VEGF) drug injections may lose the initial vision gains in vision. Protracted regular treatment schedules represent a high burden not only for patients and families, but also healthcare professionals, systems, and ultimately society too. There has been a clear need for longer-acting therapies that reduce the frequency, and therefore the burden, of treatment interventions. Several longer-acting interventions for nAMD, diabetic macular oedema, retinal vein occlusion, uveitis and glaucoma have either been developed or are in late-phase development, some of which employ novel mechanisms of actions, and all of which of promise longer ( $\geq 3$ month) treatment intervals. This review delivers an overview of anti-VEGF agents with longer durations of action, DARPins, bispecific anti-VEGF/Ang2 therapies, anti-PDGF and anti-integrin therapy, Rho-kinase inhibitors, the Port Delivery System, steroids, gene therapy for retina and uveitis, and for glaucoma, ROCK inhibitors, implants and plugs, and SLT laser and MIGS. The review also refers to the potential of artificial intelligence to tailor treatment efficacy with a resulting reduction in treatment burden.

Eye (2022) 36:1154-1167; https://doi.org/10.1038/s41433-021-01766-w

\section{INTRODUCTION}

The past two decades have seen significant advances in our ability to treat ocular disease, particularly those of the posterior segment. In particular, the advent of anti-vascular endothelial growth factor (VEGF) drugs has transformed the treatment of retinal diseases such as neovascular age-related macular degeneration (AMD), diabetic macular oedema (DMO), and macular oedema secondary to retinal vein occlusions (RVO). In glaucoma, the introduction of the prostaglandin analogue latanoprost, and more recently, the RhO-kinase (ROCK) inhibitor netarsudil and the nitric oxide (NO) latanoprostene bunod have resulted in significant therapeutic benefits for patients.

However, all of these therapies are associated with a relatively high treatment burden [1]: for example, intravitreally administered anti-VEGF therapy can involve hospital visits for assessment and anti-VEGF injections every 1-2 months. Given that many ocular diseases are age-related, and in 2019, the 'baby boomer' generation (those aged between 55 and 73 years) represented $21 \%$ of the population of the United Kingdom [2], the inevitable increase in age-related eye disease is associated with a heavy treatment burden for patients, their families, and the National Health Service.
Glaucoma is also largely a disease of an ageing population, and although the treatment is primarily topical therapy, this still requires strict compliance. It was estimated in 2020 that glaucoma represents the cause of moderate or severe visual impairment in 4.1 million people and blindness in 3.6 million [3]. Primary openangle glaucoma (POAG) accounts for two-thirds of cases, with primary angle-closure glaucoma (PACG) the next most common form of the condition [4]. Most forms of glaucoma, including secondary glaucomas, are associated with a raised intraocular pressure (IOP) as the major causative risk factor. All current treatments target IOP to control disease progression, and treatment options include medical, laser or surgical therapies. The mainstay of glaucoma therapy remains medical treatment in the form of eye drops, although accurate continued compliance to prescribed treatment regimens has been found to be suboptimal, especially when compared with other chronic medical conditions [5].

The reality is that patients have busy lives, have other commitments, and other health concerns as they age. They may forget an appointment or to take their eye drops, and with anti-VEGF therapy, may miss injections because of reluctance to be a burden to the relatives or carers who bring them to the

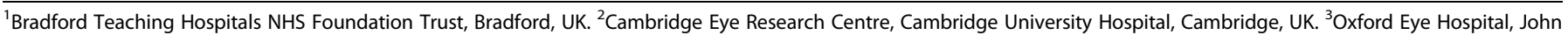

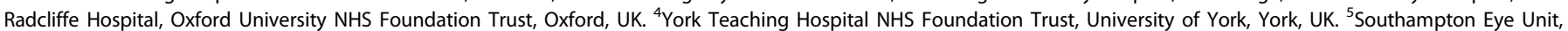

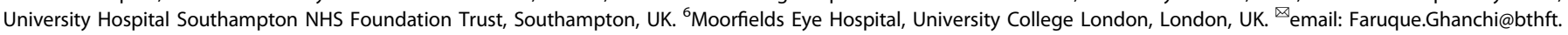
nhs.uk

Received: 20 July 2021 Revised: 19 August 2021 Accepted: 7 September 2021

Published online: 1 January 2022 
appointment, have needle-phobia, or simply become weary of repeated visits to an eye clinic. One approach that should help address these issues is the introduction of longer-acting therapies. Longer-acting therapies-particularly those that require fewer clinic visits for treatment administration-will result in a lower burden for patients (reducing the number of clinic visits means less time spent on travel and hospital appointments, with less disruption to work); for families and carers (who accompany the patient), as well as for overstretched healthcare systems. Therapies with extended durations of action will lessen the risk of forgotten or missed doses. In addition, novel treatments (e.g. gene therapies for inherited retinal disorders), will result in even greater demand for the services of eye care professionals, further increasing the pressure on already stretched health resources. The introduction of longeracting therapies is therefore welcome and timely.

For the purpose of this review article, we defined 'long-acting' therapies as those with a potential treatment effect of more than 12 weeks. We included glaucoma in this review, as longer durations of treatment effects would help resolve issues with patient compliance associated with topically administered antiglaucoma drug regimens. Innovative drug delivery methods and routes are used to achieve the goal of longer duration of action in the eye (Fig. 1).

\section{AMD, DMO, RVO AND UVEITIS}

\section{Anti-VEGF therapy}

Pegaptanib sodium (MacuGen, Pfizer, New York, NY, USA) was the first anti-VEGF agent approved for ophthalmic use but was superseded by other agents. The next anti-VEGF agent to be approved, ranibizumab, has a posology for neovascular agerelated degeneration (nAMD), DMO, PDR and RVO at one injection per month until maximum visual acuity is achieved (or there are no signs of disease activity) and thereafter, the decision when to treat with the next dose is determined by the treating physician based on disease activity, as assessed visual acuity or anatomical parameters [6], a regimen called 'treat-and-extend' (Table 1). If disease activity recurs, then the dosing interval is shortened. There are no data on the use of intravitreal ranibizumab injections at 12 or more weekly intervals. Bevacizumab (Avastin, Roche, Basel, Switzerland), which is used to treat certain cancers, is frequently used off-label as an alternative to European Medicines Agency (EMA)-approved anti-VEGFs. Bevacizumab is supplied in a singleuse vial (4 mL, $100 \mathrm{mg}$ ) for systemic use [7], and these doses are withdrawn from the vial under sterile conditions to give $1.25 \mathrm{mg}$ doses in a volume of $0.05 \mathrm{~mL}$. This approach, while timeconsuming, can be very cost-efficient for a drug that is reasonably similar in its efficacy to the other anti-VEGFs currently available $[8,9]$. There is no specific guidance for bevacizumab's posology, but its initial studies in the eye employed a monthly dosing regimen, and later studies lent support for administration in treatand-extend [10] and pro re nata (PRN) regimens [11]. However, treat-and-extend studies that compared bevacizumab to licensed anti-VEGF agents indicated a need for more frequent injections, as bevacizumab use was associated with less macular fluid resolution [12]. There are no studies comparing bevacizumab with current licensed anti-VEGF actions for a longer extension of 12 or more weeks. Aflibercept (Eylea, Bayer HealthCare, Berlin, Germany) is another anti-VEGF agent that also blocks the pro-angiogenic cytokine, placental growth factor (PIGF). Based on the evidence from Phase III trial programme [13-18], aflibercept's approved posology involves administering the drug initially as three (nAMD) or five (DMO) monthly loading doses, followed by dosing every second month for the first year (or in patients with RVO, monthly treatment [typically $\geq 3$ months] until maximal visual acuity gains

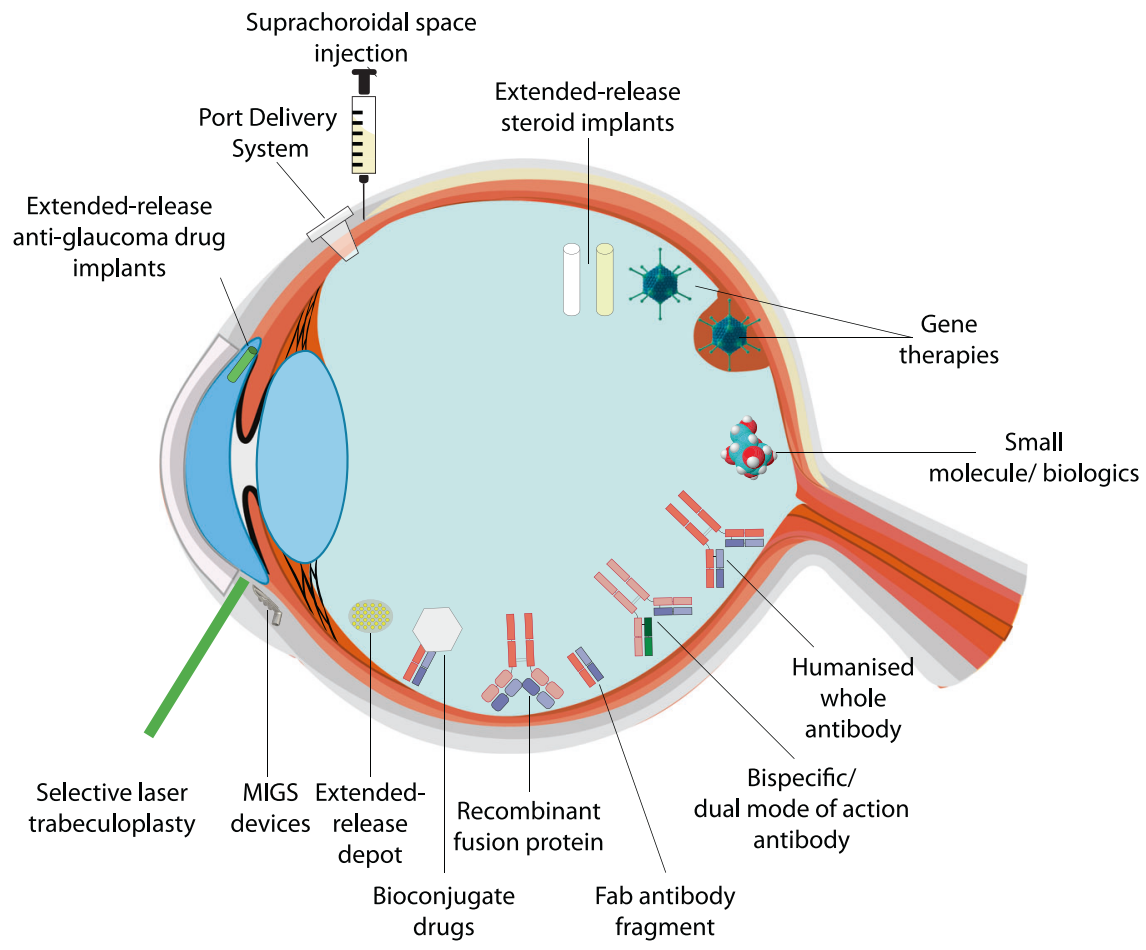

Fig. 1 Schematic illustration of the types of ocular therapeutics and interventions that can deliver long therapeutic durations of action. For retinal disorders intravitreal injections are employed to deliver various antibodies, extended release depot preparations and implants, as well as new biologics and some gene therapy. Subretinal injections (after vitrectomy) are used to deliver gene therapy, suprachoroidal injection route is employed to deliver steroids and has potential for use with other agents. Port delivery system anchored at pars plana is used as a reservoir of therapeutic agent that dissolves in vitreous over time. For glaucoma the options include extended release implants in the anterior chamber, MIGS devices and selective laser trabeculoplasty. 
F. Ghanchi et al.

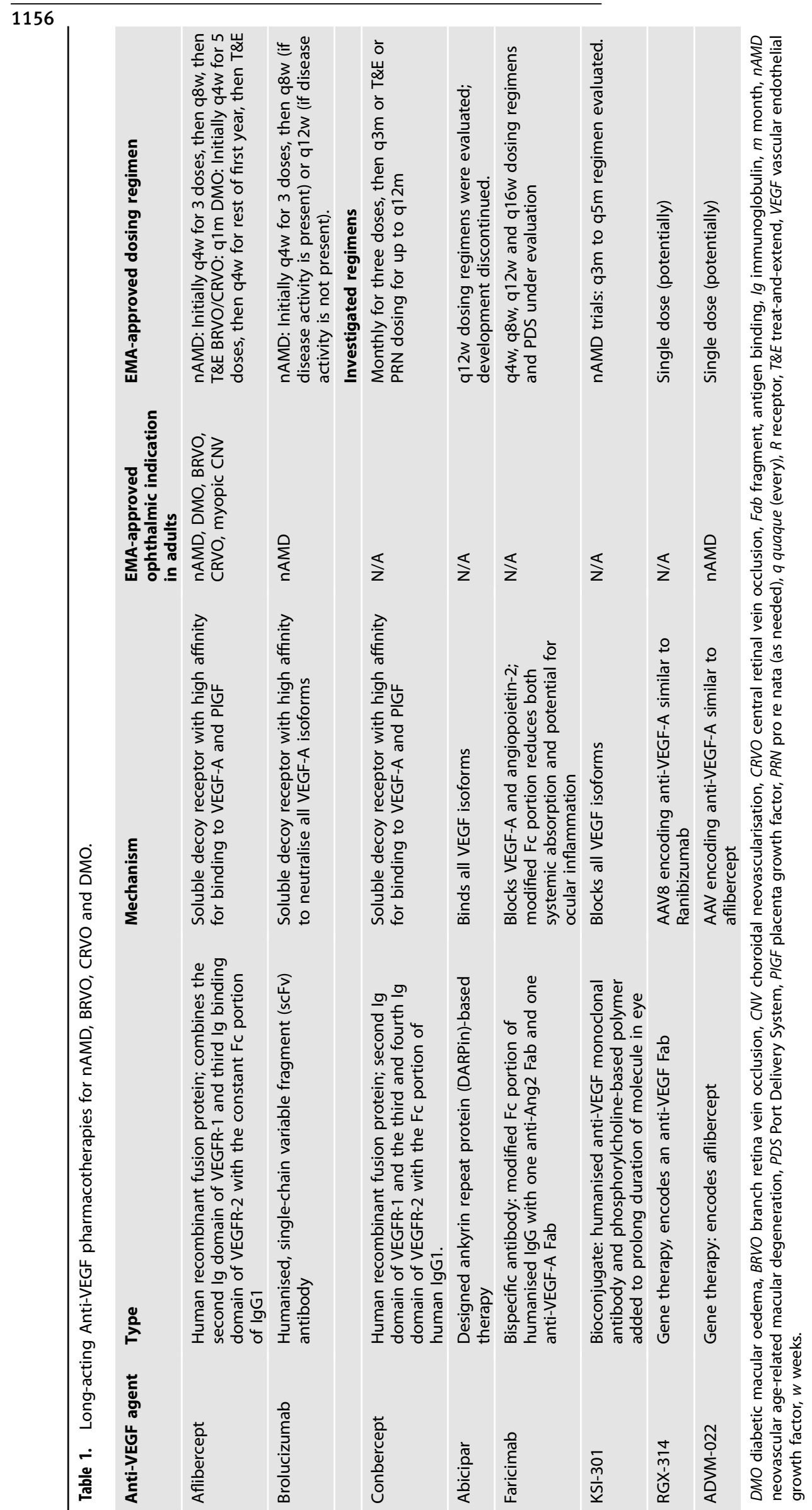


are achieved). After this point, a treat-and-extend regimen is possible [19].

\section{Anti-VEGF agents with proven longer duration of action}

Aflibercept: Recently, two studies, ALTAIR [20] and ARIES [21], investigated aflibercept for the treatment of treatment-naïve patients with nAMD, and used a treat-and-extend regimen that extended treatment out to a maximum of 16-week (i.e. 4-month) intervals. Patients in both trials were divided into two groups: those who had their treatment interval extended by 2 weeks after every assessment, or those who had it extended by 4 weeks. In the ALTAIR study, the switch to treat-and-extend was after 3 monthly aflibercept injections in a mostly Japanese population, but in the ARIES (predominantly European descent participants) study, the switch to treat-and-extend was deployed either early (after the three initial monthly doses) or late (at the 52-week stage, before then, patients received aflibercept dosing every second month). In ALTAIR, the best-corrected visual acuity (BCVA) letter gains in the 2-week and 4-week extension groups were, respectively, +9.0 and +8.4 letters at 52 weeks, and +6.1 letters at week 96 , with the last injection interval being $\geq 12$ weeks in $42.3 \%$ and $49.6 \%$ of patients, respectively, at 52 weeks, and $56.9 \%$ and $60.2 \%$, at 96 weeks, with the mean number of injections in both groups being 10.4 at this time point. The study authors noted that the 'outcomes were similar between the 2- and 4-week groups'. The ARIES study investigators found that outcomes were fairly similar in the groups where patients switched to a treat-andextend regimen early (week 104 BCVA change from baseline +4.3 letters; a mean of 12.0 injections; $47.2 \%$ of patients had last injection interval was $\geq 12$ weeks) or late $(+7.9$ letter gain; a mean of 13.0 injections; $51.9 \%$ had a last injection interval of $\geq 12$ weeks; mean of 13.0 injections).

Ziv-aflibercept (Zaltrap, Sanofi, Paris, France, indicated to treat colon cancer via systemic infusion) contains the same active molecule (aflibercept) but in a different buffer, and is supplied in 4 $\mathrm{mL} / 100 \mathrm{mg}$ and $8 \mathrm{~mL} / 200 \mathrm{mg}$ vials. Ziv-aflibercept can be prepared in a similar manner to bevacizumab for intravitreal injection, and its off-label use to treat $\mathrm{nAMD}$, macular oedema secondary to RVO and DMO, is reviewed elsewhere [22]. Zivaflibercept has been investigated in two Phase I trials with PRN dosing (NCT04290195 and NCT02173873) and is being investigated with up to 12 weekly doing in one Phase 2 trial (NCT02173873), although none of these trials have reported results to date.

Brolucizumab: In 2020, brolucizumab (Beovu, Novartis Pharma AG), another humanised monoclonal single-chain antibody fragment that targets VEGF-A, was approved for the treatment of $\mathrm{nAMD}$, and is currently undergoing late-phase clinical investigation for other retinal diseases, including PDR (NCT04278417), DMO (NCT04058067), and RVOs (NCT03802630 and NCT03810313). In nAMD, brolucizumab is administered monthly for the first three doses, thereafter the dosing regimen is decided by the treating physician based on visual acuity/anatomical parameters, with the EMA-approved posology being 'in patients without disease activity, treatment every 12 weeks ( 3 months) should be considered. In patients with disease activity, treatment every 8 weeks ( 2 months) should be considered.' The Phase III brolucizumab HAWK and HARRIER studies [23] that were instrumental in its approval for the treatment of $\mathrm{nAMD}$ showed that after three monthly loading doses, up to $50 \%$ of patients could be maintained on 12 weekly dosing intervals. It showed that as early as 16 weeks after commencement of therapy (after three injections), that it can be identified whether a patient requires 8 -weekly or 12 weekly injections. However, ocular inflammation rates of $4.6 \%$ in the HAWK and HARRIER studies (intraocular inflammation: 3.3\%, retinal vasculitis, $2.1 \% ; 0.5 \%$ of these findings were associated with severe vision loss) [23] and post-marketing reports of intraocular inflammation, in particular, retinal vasculitis and vascular occlusion are of concern and have highlighted the importance of case selection as well as vigilance [24-29]. These concerns were highlighted by the release of the 1-year data from the Phase 3 MERLIN trial (NCT03710564) that compared 4-weekly regimens of brolucizumab and aflibercept in patients with nAMD and persistent retinal fluid despite anti-VEGF therapy. This trial met its primary efficacy endpoint of noninferiority to aflibercept in terms of VA gains and certain anatomical outcomes, but again showed that brolucizumab was associated with higher intraocular inflammation rates $(9.3 \%)$ than aflibercept $(4.5 \%)$ when administered using the same regimen (retinal vasculitis: $0.8 \%$ vs. $0.0 \%$; retinal vein occlusion: $2.0 \%$ vs. $0.0 \%$ ) [30]. The rates of vision loss from any cause were $4.8 \%$ and $1.7 \%$, respectively. Following this, Novartis announced the termination of the MERLIN, branch RVO RAPTOR (NCT03802630) and central RVO RAVEN (NCT03810313) Phase 3 studies of brolucizumab and have advised Brolucizumab should not be used if less than $q 8 \mathrm{w}$ injections are needed after initial loading phase [30].

Nevertheless, multiple brolucizumab dosing regimens are currently under investigation for diabetic retinopathy in Phase 3 trials. These include three 6-weekly loading injections, followed by 12 weekly maintenance doses for PDR (NCT04278417); and for DMO, five loading doses with 'subsequent doses per protocolspecified maintenance schedule' up to 12 weekly dosing (NCT03481660; NCT04079231, NCT03481634) and monthly dosing (NCT03917472). Similarly, the TALON study (NCT04005352) is currently evaluating brolucizumab in extended treatment interval (treat-and-extend fashion) compared to aflibercept in patients with nAMD.

Conbercept: Conbercept (Lumitin, Chengdu Kanghong Biotech Co., Ltd., Sichuan, China), like aflibercept, is a VEGF receptor 1 and 2 fusion protein, which has undergone or is currently undergoing Phase III clinical evaluations for indications including nAMD, (NCT03577899, NCT03630952) DMO (NCT02194634), uveitic macular oedema (NCT04296838), and macular oedema following RVO (NCT03108352), but is currently only approved for the treatment of nAMD in China and Mongolia. The Phase III PANDA-1 trial that compared 8- and 12 weekly conbercept doses with 8-weekly aflibercept doses in patients with nAMD failed to meet its primary endpoint at 1 year (NCT03577899), and it and the PANDA-2 trial (NCT03630952) have now closed. The Phase 3 PHOENIX study, performed in patients with choroidal neovascularisation secondary to AMD, employed a dosing regimen of three monthly doses, followed by quarterly ( $\mathrm{q} 3 \mathrm{~m}$ ) dosing for the rest of the 12-month study period [31], and one Phase IV clinical trial (NCT02802657) is evaluating conbercept using both a treat-and-extend and a PRN regimen (where treatment intervals can be extended up to q12w).

Finally, another anti-VEGF agent, OPT-302 (Ophthea, South Yarra, Australia) which blocks the $C$ and D isoforms of VEGF has completed a series of Phase I and II trials in wet AMD and DMO (NCT02543229, NCT03345082, NCT03397264). Phase III trials in nAMD (NCT04757610, NCT04757636) are currently underway, but only a 4-week dosing interval is currently being investigated.

Biosimilars: It is worth noting that there are multiple anti-VEGF 'biosimilars' in development (Table 2). Their duration of action compared to the parent compound is unclear. Currently, there are no data as to whether their action can be lengthened to 12 weeks or longer. Depending on the molecule, these trials are evaluating 4- or 8-weekly dosing regimens. However, even though these agents might be cheaper than existing treatment options, the concern is that they will not be able to help with existing capacity and compliance issues if their treatment intervals are not extendable. 
Table 2. Anti-VEGF biosimilars in development ${ }^{\mathrm{a}}$.

\begin{tabular}{|c|c|c|c|}
\hline Company & Compound name & Registered clinical trials & Phase \\
\hline \multicolumn{4}{|l|}{ Aflibercept biosimilars } \\
\hline Amgen & ABP 938 & NCT04270747 & III \\
\hline Alteogen & ALT-L9 & NCT04058535 & 1 \\
\hline Coherus Biosciences & CHS-2020 & - & \\
\hline Formycon & FYB203 & NCT04522167 & III \\
\hline Momenta/Mylan & MYL-1701P & NCT03610646, NCT04674800 & III (both) \\
\hline \multicolumn{4}{|l|}{ Ranibizumab biosimilars } \\
\hline Coherus Biosciences/Bioeq & FYB201 & NCT02611778 & III \\
\hline Lupin & LUBT010 & NCT04690556 & III \\
\hline Samsung Bioepis & SB11 & NCT03150589 & III \\
\hline Xbrane & Xlucane & NCT03805100 & III \\
\hline
\end{tabular}

${ }^{a}$ Duration of efficacy to be determined.

\section{DARPins}

Abicipar pegol (Allergan/Abbvie, Dublin, Ireland), is a designed ankyrin repeat protein (DARPin)-based therapy that binds all VEGF isoforms [32]. It has a considerably longer half-life than ranibizumab in aqueous humour (13 vs. 7 days). A pooled analysis of two Phase III trials, CEDAR and SEQUOIA [33], has demonstrated its noninferiority to ranibizumab when administered at 12-week injection intervals compared with ranibizumab's 4-week intervals [34]. Issues with ocular inflammation, thought to be related to the manufacturing process of the drug, were observed in CEDAR and SEQUOIA (the 1-year incidence of intraocular inflammation was $15.4 \%$ and $15.3 \%$ for abicipar dosed every 8 and 12 weeks, respectively). The manufacturing process was modified and abicipar was evaluated in patients $(n=123)$ with nAMD over a 28-week period in the open-label MAPLE study (NCT03539549). Patients received three monthly loading doses followed by 8-weekly doses, for a total of five injections. The incidence of intraocular inflammation was reduced to $8.9 \%$ [35]. To date, abicipar has not been approved by the US Food and Drugs Administration and its clinical development remains paused.

\section{Bispecific/dual action drugs}

The bispecific antibody faricimab (Roche, Basel, Switzerland) targets not only VEGF-A, but also angiopoietin (Ang)-2. The Angtyrosine kinase endothelial receptor (Ang-Tie) pathway is responsible for regulating vascular homoeostasis through Tie-2 receptor, the breakdown of which leads to vascular permeability, inflammation, and angiogenesis. In a healthy state, Ang 1 binds the Tie-2 receptor, the pathway becomes activated, and this maintains vascular health. Under the pathological conditions found in AMD or DMO, however, Ang2 acts as a competitive antagonist of Ang1, and inhibits the activation of the Tie- 2 receptor, destabilising the retinal vasculature and making it more susceptible to the effects of pro-inflammatory cytokines and VEGF [36].

Faricimab has been evaluated in two Phase II trials (STAIRWAY [37] and AVENUE [38]) for the treatment of nAMD, and one Phase II study for the management of DMO (BOULEVARD [39]). AVENUE and STAIRWAY showed that faricimab, with either monthly dosing (AVENUE) or every 12 or 16 weeks (STAIRWAY) provided similar clinical outcomes to patients treated with 4-weekly ranibizumab [40]. The BOULEVARD study employed 4-weekly dosing, but the Phase III faricimab DMO trials (YOSEMITE, NCT03622580, and
RHINE (NCT03622593) employed an 8-12 or 16 weekly dosing interval regimen (personalised to each patient regimen) compared with 8-weekly aflibercept dosing (after completion of the 3-month, monthly injection loading phase). One-year outcome results from RHINE and YOSEMITE (for DMO) and Tenaya and Lucerne (AMD) showed that faricimab met the primary outcome measures, and showed the majority $(70 \%)$ of patients could be maintained at 12 weekly injections and nearly half could be maintained on 16 weekly dosing [41].

Another anti-VEGF/Ang2 bispecific antibody in development is BI 836880 (Boehringer Ingelheim Pharma GmbH \& Co KG, Biberach, Germany), which is currently undergoing a Phase I dose-ranging clinical trial (NCT03861234) for the treatment of nAMD. The future might see more biphasic antibodies that target disease-causing or disease-exacerbating cytokines, with the development of a platform to create dual targeting fragment antigen-binding region (DutaFab) molecules. This approach has already been used to create DutaFabs that bind both VEGF-A and platelet-derived growth factor (PDGF)-BB with high affinity [42].

\section{Bioconjugate drugs}

Another method of producing longer-acting therapies is to bind therapeutic molecules covalently to lipid or polymer carrier molecules in a process that results in 'bioconjugate' drugs [43]. One such bioconjugate, KSI-301 (KODIAK sciences, Palo Alto, CA, USA) is formed from a humanised anti-VEGF monoclonal antibody and a phosphorylcholine-based polymer, with the polymer acting to prolong the molecule's duration in the eye following intravitreal injection [44]. A Phase I dose-ranging study (NCT03790852) included patients with nAMD, DMO or RVO and utilised a dosing regimen of three initial monthly doses, followed by retreatment as per the study protocol. Preliminary results show that $92 \%$ of eyes with nAMD eyes could be extended to $\geq 3$ months after the last loading dose without receiving retreatment; in $\mathrm{DMO}, 72 \%$ of eyes could be extended to $\geq 4$ months, and in RVO, half of all eyes could be extended to $\geq 3$ months [45]. A Phase II trial, DAZZLE (NCT04049266) for patients with nAMD is currently ongoing where 12-, 16-, and 20-week treatment intervals are under investigation. Seventy-five percent or more patients could be maintained at 16 weekly intervals at 1 year, while 2 out of 3 patients across the three indications had treatment-free intervals of 6 months or more [46]. 


\section{Kinase/cytokine inhibitors}

Tyrosine kinases are a family of enzymes that phosphorylate and activate numerous receptors. Tyrosine kinase inhibitor (TKI) drugs can therefore inactivate certain receptors (e.g. VEGF receptors) that are responsible for driving the pathologies involved in many retinal diseases. One such TKI is sunitinib maleate, which has activity against both VEGF-A and PDGF and is being developed as GB-102 (GrayBug Vision, Inc., Redwood City, CA, USA) for use as an nAMD and DMO therapy. The drug has an extended-release formulation; sunitinib is encapsulated within bioerodable and slowly degradable polymer nanoparticles designed to release clinically effective sunitinib concentrations over a 6-month period, meaning the drug can be administered by intravitreal injection every 6 months [47]. Results from the nAMD Phase I/Ila study, ADAGIO, showed that GB-102 remained effective in $88 \%$ and $68 \%$ of patients at 3 and 6 months after dosing, respectively [48]. ALTISSIMO, a Phase II trial in nAMD (NCT03953079), is currently ongoing and results are expected in 2021. Finally, a Phase II trial in patients with DMO or RVO (NCT04085341) completed in July 2020.

OTX-TKI (axitinib intravitreal implant, Ocular Therapeutix Inc., Bedford, MA, USA) is a dried polyethylene glycol-based hydrogel fibre-containing dispersed microcrystals of the small molecule tyrosine kinase inhibitor axitinib, which is designed to deliver therapeutic concentrations of the drug over the course of a year in order to enable 12-monthly dosing in patients with nAMD. Axitinib inhibits VEGF1-3, c-KIT and the PDGF receptor. Preliminary findings from a Phase I trial of OTX-TKI presented at the 2020 Retina Society meeting suggested that the durability of therapy 'was up to 4.5 months' and that the 'implant biodegraded in all subjects in cohort 1 by 9-10.5 months' [49]. The OASIS trial (NCT04626128) is a Phase I/II open-label, dose-escalation study looking at axitinib suspension injections in suprachoroidal space, and results are due to be available in late 2021. Finally, PanOptica Pharma (Mount Arlington, NJ, USA) is developing a topically applied TKI drop, PAN-90806, which reaches the retina via the trans-scleral vascular route to reach target tissues. It has been investigated in Phase I/II clinical trials for the treatment of nAMD (NCT03479372, NCT02022540) and proliferative diabetic retinopathy (NCT02475109). Nevertheless, its potential role, either as monotherapy or perhaps as an adjunctive therapy that might help reduce treatment intervals with other agents, still needs to be confirmed future studies.

\section{Anti-PDGF therapy}

Several of the drugs mentioned above target PDGF, and this action is important, as the response to anti-VEGF therapy can wear off over time. This is because the new blood vessels start to become covered with pericytes that protect the new vessels and nourish them with several cell survival cytokines, including VEGF. PDGF is one molecule that recruits the pericytes to the vessels, and thus blocking PDGF could improve outcomes by enhancing the effect of anti-VEGF. Anti-PDGF therapy has been tried in combination with ranibizumab in the past as a naïve $\mathrm{nAMD}$ therapy. A 32-mer pegylated DNA aptamer that binds PDGF-BB and PDGF-AB homodimers and heterodimers agent pegpleranib (Fovista, Ophthotech, New York, NY, USA) was evaluated in a pair of Phase III trials (NCT01944839 and NCT01940900) but ultimately failed to show any superiority over ranibizumab alone [50]. Nevertheless, there is a solid mechanistic rationale for PDGF inhibition, and it could be that PDGF inhibition, in combination with the inhibition of VEGF and other cytokines, might be a strategy that may eventually prove its worth.

\section{Recombinant, human complement factor $\mathbf{H}$ therapy}

GEM103 (Gemini Therapeutics, Cambridge, MA, USA) is a recombinant human complement factor $\mathrm{H}$ (CFH) molecule that is currently under Phase II clinical trial investigation for both neovascular (NCT04684394) and dry AMD (NCT04643886) using monthly and every other month dosing regimens. CFH plays a central role in regulating the immune system's alternative pathway in terms of protecting host cells during an inflammatory/immune response. Over-activation of the innate immune system is thought to be one of the factors that can lead to the pathology in dry AMD. Lampalizumab (Roche) was a humanised Fab that binds to complement factor D, and 4-weekly and 6-weekly dosing regimens were under investigation in Phase III clinical trials for the treatment of geographic atrophy (GA; NCT02745119, NCT02247531, and NCT02247479). However, the trials were terminated due to failure of the drug to meet the trial's primary endpoints.

Pegcetacoplan (Apellis Pharma, Waltham, MA, USA) a synthetic peptide-polyethylene glycol polymer conjugate that binds and inhibits complement proteins C3 and C3b. It is currently under Phase III clinical investigation for the treatment of GA secondary to AMD (NCT04770545, NCT03525600) using 4-weekly and 8-weeky dosing regimens. Twenty-four-month data from an earlier Phase 1b APL2-103 (NCT03777332) in 13 patients (where the eye with the worst BCVA received the study drug) revealed that GA lesions pegcetacoplan-treated eye had a growth rate that was $46 \%$ lower than the untreated fellow eye $(p=0.007 \%)$ [51]. The results of the larger Phase III DERBY (NCT03525613) and OAKS (NCT03525600) trials, are expected in the third quarter of 2021 [51].

\section{Integrin-targeting therapy}

Risuteganib, (Luminate, ALG-1001, Allegro Ophthalmics, San Juan Capistrano, CA, USA), targets integrins av $\beta 3$, av $\beta 5$, and $\alpha 5 \beta 1$, all of which are receptors involved with angiogenesis. Risuteganib has completed four Phase 3 trials: one for dry AMD (NCT03626636); one for nAMD (NCT01749891), one for DMO (NCT02348918) trials; and one for the treatment of vitreomacular adhesion (NCT02153476). Results have been published for the DMO trial, DEL MAR, in which four doses of risuteganib were compared with bevacizumab $[52,53]$. Risuteganib was administered at weeks 0,4 and 8, with an asneeded further injection at week 20. Bevacizumab was administered every 4 weeks for the first three doses, then as-needed at weeks 12 , 16 , and 20 . The primary endpoint of noninferiority compared to bevacizumab in terms of BCVA and improvement in central macular thickness was met at the 20-week time point. Allegro therapeutics had announced a Phase III trial of risuteganib in 2018, which are expected to start in late 2021/ early 2022.

\section{Rho-kinase inhibitors}

Rho-kinase inhibition has become an attractive therapeutic target in ophthalmology. The upregulation of the Rho-kinase (ROCK) pathway has been shown, in diabetes, to promote angiogenesis and vasculopathy, thus inhibiting ROCK signalling could have beneficial effects in treating neovascular diseases of the retina, either alone, or in combination with other therapies. It also has a role in glaucoma therapy: netarsudil (Aerie Pharmaceuticals, Inc., Irvine, CA, USA) is a ROCK inhibitor marketed in Europe as Rhokiinsa, (and Rhopressa in North America) that is available as a $0.02 \%$ ophthalmic solution that is used to lower the IOP in ocular hypertension or open-angle glaucoma [54]. In this context, ROCK inhibition appears to both increase aqueous humour outflow through the trabecular meshwork and reduce venous pressure in the episcleral layer of the eye. Netarsudil is a prodrug and after topical administration, it is rapidly metabolised to its active metabolite, AR-13503 by esterases in the cornea [55]. AR-13503, formulated in a biodegradable sustained release implant that releases the drug over a 4-6 month period, is under early phase clinical investigation by Aerie as monotherapy for $\mathrm{nAMD}$ and DMO (NCT03835884). Another ROCK inhibitor, Fasudil (HA-1077, Asahi Kasei Pharma, Tokyo, Japan) has shown promise when administered in combination with bevacizumab in a pilot study performed in patients with DMO [56]. 
1160

\section{Others}

It has been observed that synonymous single nucleotide polymorphisms (SNPs) in the gene, high-temperature requirement A1 (HtrA1) increase the inherited risk of nAMD [57]. HtrA1 encodes a serine protease, and recently a potent anti-HtrA1 Fab inhibitor of HtrA1's proteolytic activity, Fab15H6.v4.D221, has been developed, and a substrate (and biomarker) of HtrA1 activity, Dickkopfrelated protein 3 (DKK3), has also been identified [58]. A small Phase 1 clinical study of the antibody in patients with GA secondary to AMD showed dose-dependent inhibition of DKK cleavage that lasted for over 8 weeks following a single intravitreal Fab15H6.v4.D221 injection [58].

\section{Implants}

Port delivery system (PDS). A different method of delivering effective drug doses over an extended period is to use a slowreleasing intraocular device such as the PDS device (Roche). The PDS is a permanent, refillable implant that is placed in the eye through a small incision in the sclera and pars plana. The PDS has a self-sealing septum in the centre of the implant flange which allows clinicians to refill the implant reservoir. The drug present in the PDS passively diffuses along a concentration gradient from the implant reservoir to the vitreous cavity, via a porous metal release control element. This approach is currently under investigation with not only ranibizumab [59], but also faricimab (NCT04567303) -and it is possible that the PDS refilling interval will be approximately 4 months or longer. The Phase III ARCHWAY study (NCT03677934) evaluated ranibizumab in patients with nAMD, administered monthly (at a concentration of $10 \mathrm{mg} / \mathrm{mL}$ ) by intravitreal injection, or with the PDS implant filled with ranibizumab at a concentration of $100 \mathrm{mg} / \mathrm{mL}$. It was reported that $98.4 \%$ of patients in the PDS group were able to go 6 months without needing rescue treatment and at weeks 36-40, achieved similar mean BCVA gains to patients receiving monthly ranibizumab eye injections ( 0.2 and 0.5 letter gain from baseline, respectively) [60]. Results from Phase II of the LADDER trial of the PDS with ranibizumab for wet AMD showed comparable outcomes over 22 months with monthly intravitreal injections of the drug $[59,61]$. A new Phase IIIb study (NCT04657289; not yet recruiting) aims to compare 24- and 36-week refill regimens for the PDS with ranibizumab, thus further decreasing the treatment burden on patients and improving medication adherence.

Corticosteroid treatments. Corticosteroids are a powerful tool for controlling ocular inflammation and act by reducing the expression of a wide range of pro-inflammatory and pro-angiogenic cytokines (including VEGF), and have successfully been used in treating DMO and uveitis. Their use in DMO is typically second-line to anti-VEGF therapy, as intravitreal steroid application can be associated with increases in IOP in some patients and accelerated cataract development in most patients who still have their natural crystalline lens [62], and anti-VEGF use is still the default for the subset of patients who may need to avoid steroid therapy. Recently, an expert panel established a consensus on the management of DMO using dexamethasone implants, favouring their utility in a variety of patient situations (pseudophakic, poorly adherent, candidates for cataract surgery, high inflammatory component, history of cardiovascular events, and more) [63]. Regular intravitreal injections can present adherence challenges for patients, thus the introduction of intravitreal implants offers a longer-lasting and potentially a more convenient option. They also demonstrate superiority in vitrectomised eyes, in which VEGF inhibitors are more rapidly cleared [64]. Patients who fail VEGF inhibitor therapy have shown functional and anatomic improvement after switching to steroid therapy [65].

Dexamethasone implant: An extended-release intravitreally implanted formulation of dexamethasone (Ozurdex, Allergan/
Abbvie, Irvine, CA, USA) was approved by the European Medicines Agency for the treatment of DMO in pseudophakes or patients who are considered insufficiently responsive to, or unsuitable for non-corticosteroid therapy, macular oedema following either branch or central RVO, or inflammation of the posterior segment of the eye presenting as non-infectious uveitis [66-69] The retreatment interval varies by indication: in $\mathrm{DMO}$, it is recommended that retreatment is performed after ' 6 months', whereas retreatment in patients with RVO or uveitis 'should be considered when a patient experiences a response to treatment followed subsequently by a loss in visual acuity and in the physician's opinion may benefit from retreatment without being exposed to significant risk.' In practice, clinicians use clinical markers of activity (recurrence of oedema and/or vision loss) to determine whether retreatment with dexamethasone implant is necessary, and that can be every 3-4 months.

Fluocinolone implant: A second steroid-releasing intravitreal implant, in this case, one that contains fluocinolone acetonide (lluvien, Alimera Sciences, Alpharetta, GA, USA) is also available, and is reported to release fluocinolone acetonide for a period of up to 36 months. Its approved indications in Europe are the treatment of vision impairment associated with chronic DMO considered insufficiently responsive to available therapies' and the 'prevention of relapse in recurrent non-infectious uveitis affecting the posterior segment of the eye.' [70] Retreatment intervals 'may be administered after 12 months if the patient experiences decreased vision or an increase in retinal thickness

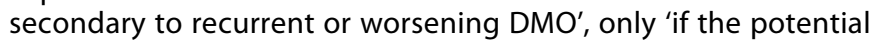
benefits outweigh the risks.' [70] Its effects on uveitis [71-73] and DMO [74, 75] have been well characterised in late-phase clinical trials.

Suprachoroidal administration of triamcinolone acetonide: Suprachoroidal administration of triamcinolone acetonide is associated with the drug diffusing through the suprachoroidal space, which concentrates the drug mostly in the sclera, choroid and retina, and minimises the amount of drug that accumulates in the anterior chamber, lens and vitreous [76, 77] an approach that should help minimise the well-known off-target steroid adverse effects of cataract formation and IOP rises. Access to the suprachoroidal space has become easier with the development of microneedles that enable drug delivery by ophthalmologists in an outpatient setting, as opposed to sclerotomy or $a b$ interno surgical approaches that were previously required [77].

A propriety, preservative-free formulation of triamcinolone acetonide for suprachoroidal administration, CLS-TA (XIPERE, Clearside Biomedical, Alpharetta, GA, USA), has undergone latephase clinical evaluation for the treatment of DMO, and macular oedema secondary to non-infectious uveitis and RVO.

A Phase III trial of CLS-TA comparing CLS-TA in combination with aflibercept with aflibercept monotherapy was performed in 460 patients with RVO and macular oedema (SAPPHIRE, NCT02980874) was terminated after 8 weeks as no additional benefit of the combination therapy was seen at that point [78]. After this, Clearside Biomedical reported that they would discontinue the clinical development of CLS-TA for RVO and focus on its development as an uveitis monotherapy [78].

A 12 weekly dosing of CLS-TA (or sham treatment, randomised in a $3: 2$ ratio) was evaluated in 160 patients with macular oedema secondary to non-infectious uveitis for a 24-week period in the Phase III PEACHTREE trial (NCT02595398). Suprachoroidally administered CLS-TA was significantly better than sham treatment at achieving $\geq 15$ letter BCVA gains from baseline ( $47 \%$ vs. $16 \%, p<$ 0.001 ) and mean reductions in central subfield thickness (CST) from baseline $(153 \mu \mathrm{m}$ vs. $18 \mu \mathrm{m}, p<0.001)$. Steroid-associated elevations in IOP rates and cataract development rates were 11.5 and $15.6 \%$, and 7.3 and $6.3 \%$ [79]. 


\section{Steroid-sparing anti-inflammatories in uveitis}

Many patients with non-infectious uveitis affecting the posterior segment will receive systemic corticosteroid therapy, particularly in the acute setting. This can be very effective, but typically multiple systemic side effects accumulate as the duration of therapy lengthens. Local steroid treatment as described above can become an option for some patients, but immunosuppressant drugs are also an option that can help reduce long-term corticosteroid dependence. These agents include antimetabolites, T-cell inhibitors, alkylating agents, and biologics targeting B- and T-cell activation, interferon therapy, and the use of drugs that interfere with the action of interleukin-6. Many of these therapies require frequent dosing and so are outside the scope of this review, but some require infrequent administration, for example, rituximab (MabThera, Rituxan, Roche) and alemtuzumab (Campath/Lemtrada, Sanofi, Paris, France).

Rituximab is a chimeric monoclonal antibody which blocks CD20, a cell surface marker found on B-lymphocytes [80]. Rituximab is commonly administered initially as two intravenous doses given 2 weeks apart, then approximately every 6 months, depending on the initial response to therapy and the recurrence of disease activity. Most clinical experience with rituximab is derived from its use in patients with rheumatoid arthritis, and these data suggest the risk of a serious infusion reaction is $<1 \%$, and infection rates have been estimated at around 2-3\% [80]. However, the evidence base for its efficacy in uveitis is more limited with only a series of case reports [81, 82], and two Phase $\mathrm{I} / \mathrm{II}$ trials for the treatment of orbital inflammation and refractory scleritis $[83,84]$. Of the 10 patients enroled in the orbital inflammation trial, seven showed improvement with rituximab infusions [84], and of the 12 involved in the scleritis, eight responded to the therapy [83]. Rituximab has also been used off-label for the treatment of retinal vasculitis or myelitis in patients with Behcet's disease [85-88].

Alemtuzumab is a monoclonal antibody that binds CD52 (a protein that is expressed on mature lymphocytes) and targets these lymphocytes for destruction. Its principal indications are for the treatment of B-cell chronic lymphocytic leukaemia, and as a thirdline treatment for relapsing-remitting multiple sclerosis [89]. Alemtuzumab is parenterally administered as daily infusions: daily for 5 consecutive days for the first dose, then daily for 3 consecutive days for the second and subsequent doses. However, the interval between each course of infusions is 12 months [89]. The evidence base for alemtuzumab in uveitis is limited; with a case report of its intravenous administration in a 17-year-old patient with active relapsing multiple sclerosis and bilateral optic neuritis (and subsequently bilateral intermediate uveitis and secondary macular oedema). The intraocular inflammation, previously refractory to conventional immunosuppressants, responded to alemtuzumab, inducing remission [90]. A clinical trial that involved 334 people with relapsing-remitting multiple sclerosis were randomised to receive either subcutaneous interferon beta-1a (IFNB-1a), alemtuzumab 12 $\mathrm{mg}$ or alemtuzumab $24 \mathrm{mg}$ [91]. Visual contrast sensitivity assessments were performed on all eyes at baseline, and every 3 months after treatment up to 36 months of follow-up. At 3 months, alemtuzumab-treated patients were significantly more likely $(p=$ 0.013 ) to experience visual improvement of at least 0.3 log units, and at the end of the follow-up period, the contrast sensitivity gains (pooled alemtuzumab: 0.080 log unit gain vs. IFNB-1a 0.038 log unit gains) were still present $(p=0.0102)$.

However, these therapies are not licensed in the United Kingdom, nor are they funded for use in the National Health Service, and it is important to note that in 2018, alemtuzumab was subject to a FDA Safety Announcement, that noted 'rare but serious' instances of stroke and blood vessel wall tears in patients with multiple sclerosis, some of which were fatal, and most of which occur within 1 day of treatment initiation [92]. In 2019, the EMA's Pharmacovigilance Risk Assessment Committee started a review of alemtuzumab following new reports of immune- mediated conditions and of problems with the heart and blood vessels with this medicine, again including fatal cases [93].

Gene therapy. Gene therapy holds considerable and transformative potential for the development of therapies that could significantly reduce treatment burden. For example, gene therapy can potentially be used to generate long-term therapeutic biological molecule production in the eye. The complexity of retinal disease pathogenesis can make the therapy more challenging to be administered, and for treatment to be successful, several factors need to be addressed including the definition of the therapeutic window, safe and efficient vectors, identification of a suitable target gene, and a reliable means of regulating transgene expression, as well as patient selection and outcome measurement.

RGX-314 is a one-time, subretinal gene therapy that uses an adenoviral vector to introduce a monoclonal antibody fragment into the eye. The antibody neutralises VEGF to reduce or eliminate abnormal blood vessel growth, has been shown to reduce anti-VEGF treatment burden, and appears to be well-tolerated as a subretinal injection [94]. The Phase II AAVIATE trial (currently recruiting) will randomise patients to receive either RGX-314 in the suprachoroidal space or control treatment (ranibizumab), thus evaluating the efficacy of a potential one- or two-dose treatment for nAMD [95]. ADVM-022 (Adverium Biotechnologies Inc., San Francisco, CA, USA) utilises a propriety vector capsid, AAV.7m8, which carries an aflibercept coding sequence under the control of a proprietary expression cassette, and preliminary results of the OPTIC trial (NCT03748784) of two doses of ADVM-022 in patients with nAMD suggest that stable aflibercept production is present at up to 30 months post-administration, with the $14 / 15$ patients who received the higher ADVM-022 dose required no rescue anti-VEGF rescue therapy (six patients with 84 weeks follow-up; six patients with 16 weeks follow-up) [96].

GT005 is another potential AAV-2 based gene therapy, this time for dry AMD. It is delivered as a one-time treatment into the suprachoroidal space in patients with GA secondary to dry AMD. GT005 upregulates complement factor I (CFI), which counters inflammation caused by an overactive complement system. The HORIZON trial (currently recruiting) will randomise patients to receive either GT005 (medium or high dose) or no treatment, thus evaluating the efficacy of a potential single-dose treatment for dry AMD [97]. The open-label Phase I/II FOCUS study recently has reported a sustained increase in CFI with single suprachoroidal delivery of GT005 [98].

\section{Artificial intelligence}

The combination of the plethora of treatment options under development, and the fact that many of them have novel (and even multiple) mechanisms of action can make choosing the optimal treatment option for patients challenging. There is much interest in the potential application of artificial intelligence (AI) in terms of automated diagnostic screening. Further to the benefits that this will bring, an additional key step in optimising treatment in these different conditions will be to integrate the prediction of the best personalised treatment plans by analysing response to agents used, evaluating dosing regimens in big datasets. This will enable us to achieve the best possible outcomes with the lowest patient and healthcare system burden [99].

\section{Glaucoma}

Glaucoma is a common, currently irreversible cause of vision loss, characterised by optic nerve head excavation and loss of visual field. The mainstay of glaucoma therapy remains medical treatment in the form of eye drops, although adherence and persistence (i.e. continued correct compliance) have been found to be suboptimal, even when compared with other chronic medical conditions. As a result, longer-acting therapies for glaucoma are of significant interest (Table 3). 


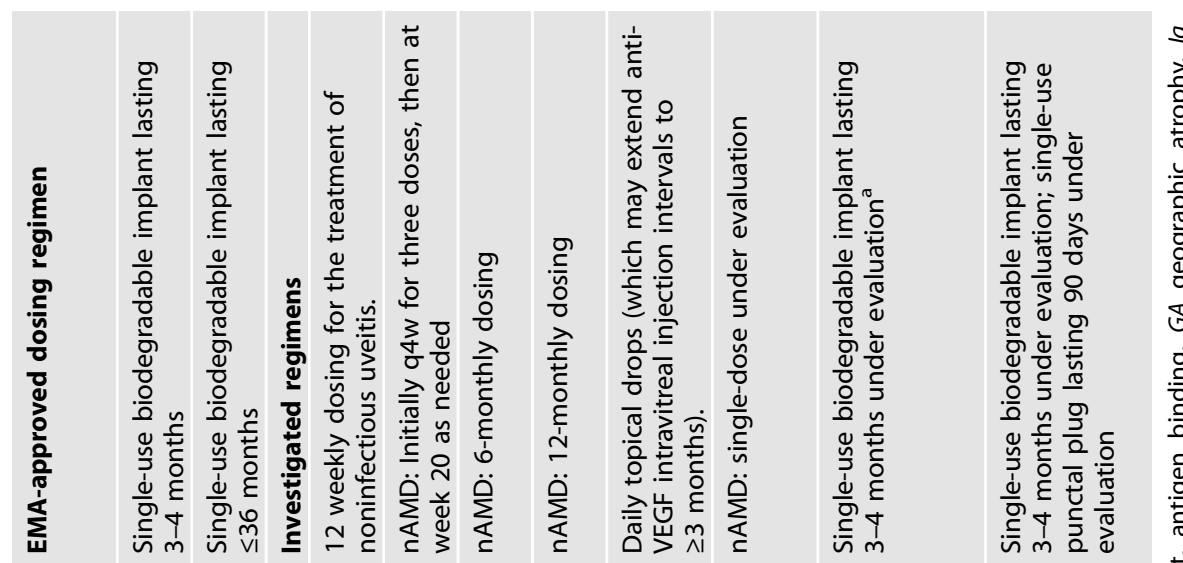

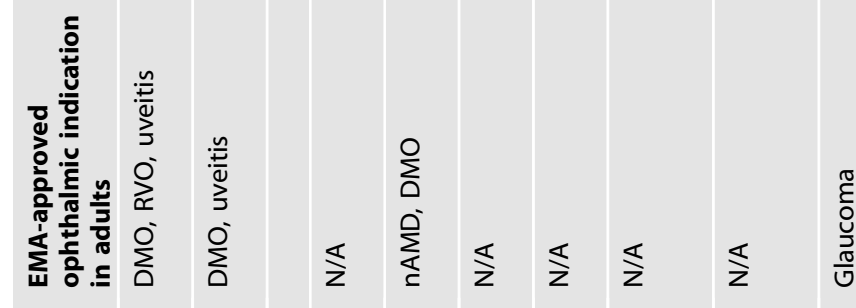

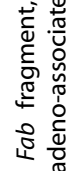

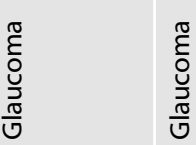

亭
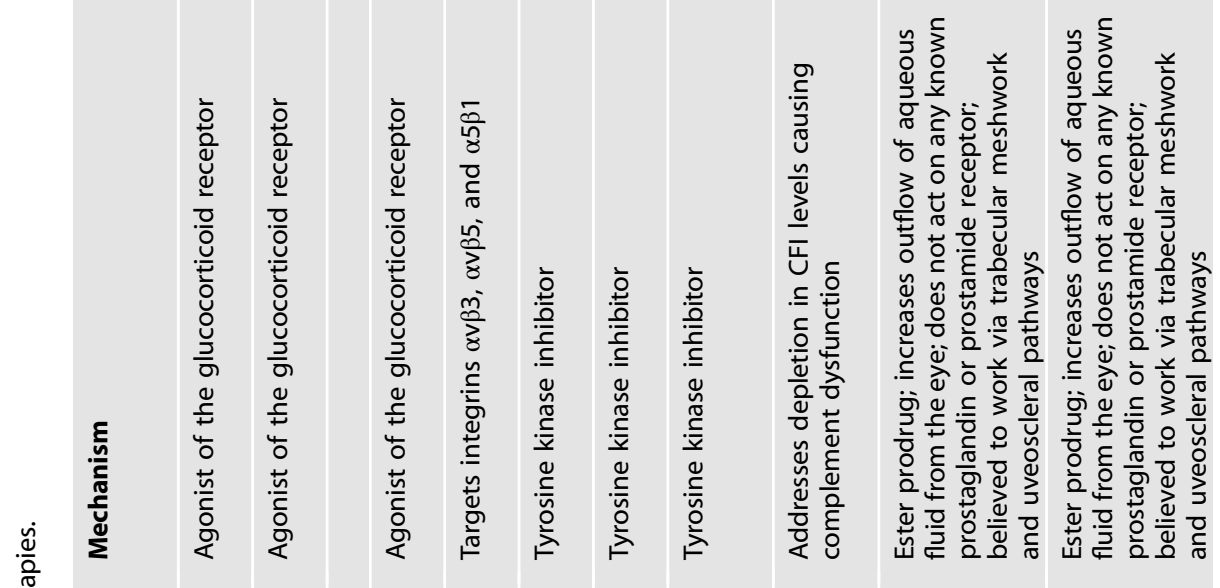

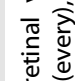

ऽ ध

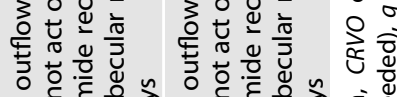

○े

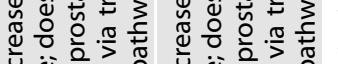

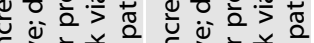
苞高
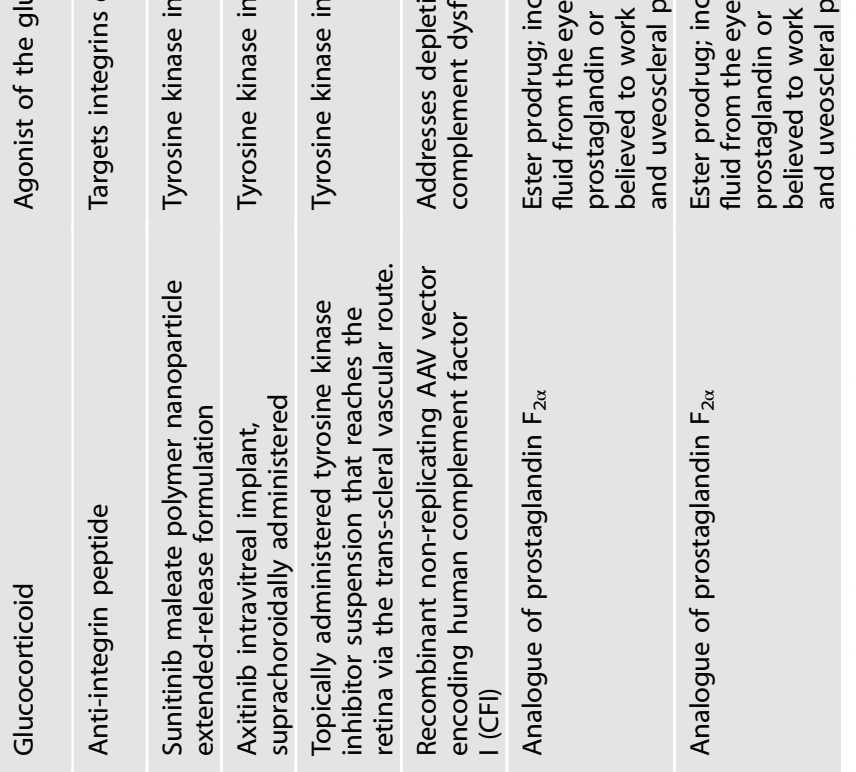

ऐ

बं

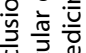
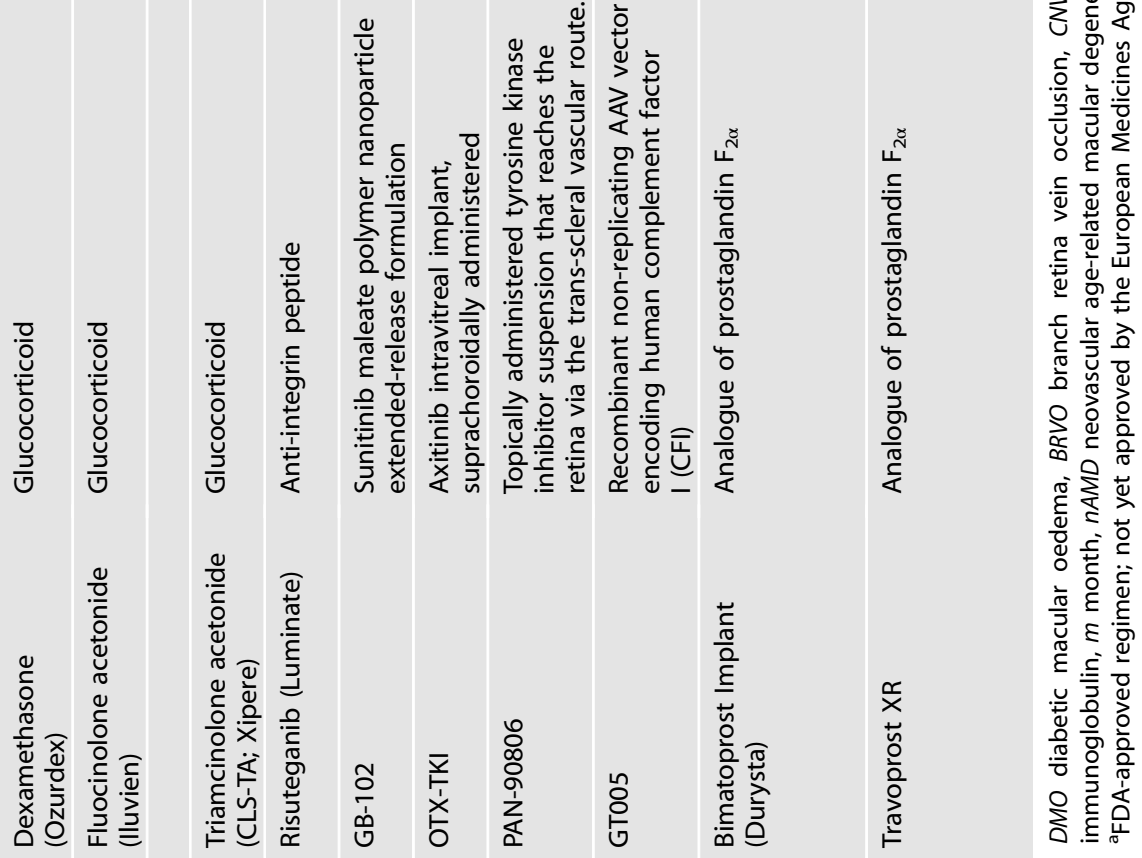

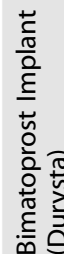

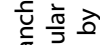

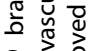

究

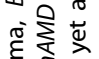

要主

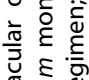

实

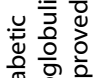

읗 흥 흥

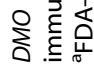


One approach has been the development of biodegradable implants, such as Bimatoprost SR (Durysta; Allergan, Dublin, Ireland) [100-102]. This utilises the Novadur (Allergan, Dublin. Ireland) biodegradable drug-delivery system which has been used as a delivery system for dexamethasone (Ozurdex; Allergan, Dublin, Ireland) since 2009 [103]. The rod-shaped implant is administered with a single-use 28-gauge applicator and provides a non-pulsatile, continuous release of bimatoprost for between 3 and 4 months [102]. Initial IOP-lowering data came from a 24month Phase I/II clinical trial, APOLLO, which found comparative IOP-lowering at a range of doses when compared to once-daily topical bimatoprost $0.03 \%[100,101]$. Later Phase III trials, ARTEMIS- 1 and ARTEMIS-2, assessed the efficacy and safety of 10- and $15 \mu \mathrm{g}$ implants in adults with either POAG or ocular hypertension $(\mathrm{OHT})$ with open inferior angles and baseline IOP of 22-32 $\mathrm{mmHg}$ after a washout period [102]. Both tested doses were non-inferior to twice-daily topical timolol maleate $0.5 \%$ at 12 weeks. In ARTEMIS-1, at 52 weeks follow-up (after administration at three fixed 16-week intervals), $84.3 \%$ (167/198) of subjects receiving the $10 \mu \mathrm{g}$ implant had not required further IOP-lowering treatment [102]. The FDA-approved Durysta in March 2020 for use in patients with POAG or OHT, currently as a one-off treatment given the potential risk for corneal endothelial cell loss.

Travoprost has also been utilised in drug-eluting intracameral implants. Travoprost XR (Aerie Pharmaceuticals, USA) is a biodegradable implant that uses novel sterile nanoparticle replication engineering technology to provide continuous release of travoprost. A 12-month study of 15 patients with POAG demonstrated noninferiority to twice-daily timolol maleate $0.5 \%$ at 11 -month follow-up, with a mean IOP reduction of $6.7 \pm 3.7 \mathrm{mmHg}$ [104]. The OTX-TIC implant (Ocular Therapeutix, USA) contains micronised travoprost released over 4-6 months. Initial Phase I trial data with 6-month follow-up showed a greater IOP-lowering effect compared to the fellow eye treated with once-daily topical travoprost [105]. An ongoing study of additional cohorts is underway to provide longer-term data. The iDose Travoprost implant (Glaukos, San Clemente, CA, USA) is a titanium intracameral delivery system that is anchored to the trabecular meshwork. The implant is encased in a membrane that provides continuous drug elution and can be replaced for ongoing treatment. A Phase II study found a similar IOP-lowering effect for two different elution rates compared with timolol maleate $0.5 \%$ at 12 -week follow-up (NCT02754596). A Phase III trial has a primary completion date of June 2021.

A travoprost eluting punctal plug, OTX-TP (Ocular Therapeutix, USA) is a hydrogel rod that swells to fit the canalicular space and continuously releases travoprost over a 90 -day period. A placebocontrolled multicentre Phase III trial found OTX-TP treated eyes had statistically significant IOP reduction in eight of nine time points over 20-weeks of follow-up, with only transient and minor adverse effects, most commonly dacyrocanaliculitis [106]. However, the system is reportedly no longer under development [107]. Other external routes recently considered have included topical forniceal inserts [108], subconjunctival injections [109], and micelle-laden contact lenses to achieve sustained drug release [110].

Longer-term IOP-lowering can also be achieved by laser treatment, most commonly as selective laser trabeculoplasty (SLT). First approved by the FDA in 2001, today SLT is widely used, more recently also as a first-line treatment option following the publication of the LiGHT study in 2019 [111]. As SLT uptake becomes more prevalent, more focus has been given to patient selection and duration of effect. A multicentre real-world analysis of 831 SLT-treated eyes found treatment success in 70\%, $45 \%$ and $27 \%$ of treated eyes at 6-, 12 - and 24-month follow-up respectively [112]. Pre-treatment IOP was found to be the most significant predictive factor for success, in line with findings in previous studies [112-114]. The SALT trial found post-procedure topical ketorolac $0.5 \%$ or prednisolone $1 \%$ may improve the IOP-lowering effect at 12 weeks follow-up, although longer-term effects require further investigation [115].

Finally, over the past decade, a series of novel surgical approaches to glaucoma have aimed to provide long-acting alternatives to topical medications for glaucoma, called minimally invasive glaucoma surgeries (MIGS). MIGS were intended to bridge the gap between medical or laser therapy and more invasive filtering surgery in mild-to-moderate glaucoma. They are meant to have a favourable safety profile ensuring prompt postoperative recovery and a reliable (but more modest) IOP reduction than that of traditional filtering surgery. Given that most of these devices have been introduced within the last 5 years, there is a lack of long-term data regarding their effectiveness, with most published evidence being limited to nonrandomised studies and uncontrolled retrospective comparisons, with few high-quality randomised controlled trials.

Longer-acting glaucoma treatments have the potential to address well-known adherence and persistence issues, reduce burden and cost on healthcare providers and remove the need for daily dosing for patients with glaucoma. More widespread usage of SLT appears likely going forward, given its low adverse effect profile. Intracameral drug-eluting systems hold promise, although concerns over damage to the corneal endothelium persist.

\section{CONCLUSION}

Many new long-acting treatment options are currently in the pipeline, and although not all molecules will make it to the market, it is likely that in the next 5 years a number will do so, and will offer the promise of 12 weekly or longer treatment intervals, translating to around four treatments per year for patients. This reduced treatment burden is clearly going to benefit patients, healthcare providers and healthcare systems-and ultimately society too. Gene therapy holds a real potential to provide longterm benefits for patients, although there are several technical and cost hurdles that need to be overcome before this approach becomes widely available. We also need to address the fact that fewer clinic visits for treatment can also mean fewer opportunities for monitoring disease progress-particularly in fellow eyes-and also for recognising any ocular side effects that might be associated with these new agents. This is an issue that may be dealt with by creating imaging hubs or self-monitoring devices to provide regular assessments. It is anticipated that this would be supported by $\mathrm{Al}$ implementation in reading centres to assess the risk of disease activity or progression and treatment would be tailored accordingly. The advent of longer-acting therapies brings new treatment options to the horizon, many of which employ novel mechanisms of action. Finally, in addition to improving patients' regimen compliance, the personalisation of these longacting treatments to patients' own specific disease biomarkers should allow better vision outcomes to be achieved, all with a lower treatment burden for all.

\section{REFERENCES}

1. Sivaprasad S, Oyetunde S. Impact of injection therapy on retinal patients with diabetic macular edema or retinal vein occlusion. Clin Ophthalmol. 2016;10:939-46.

2. Aegon. Baby Boomers Hotspots Report. 2019. https://www.aegon.co.uk/ content/dam/ukpaw/hidden/baby-boomer-report-2019.pdf. Accessed $29 \mathrm{Apr}$ 2021.

3. Flaxman SR, Bourne RRA, Resnikoff S, Ackland P, Braithwaite T, Cicinelli MV, et al. Global causes of blindness and distance vision impairment 1990-2020: a systematic review and meta-analysis. Lancet Glob Health. 2017;5:e1221-34.

4. Tham YC, Li X, Wong TY, Quigley HA, Aung T, Cheng CY. Global prevalence of glaucoma and projections of glaucoma burden through 2040: a systematic review and meta-analysis. Ophthalmology. 2014;121:2081-90. 
5. Yeaw J, Benner JS, Walt JG, Sian S, Smith DB. Comparing adherence and persistence across 6 chronic medication classes. J Manag Care Pharm. 2009;15:728-40.

6. European Medicines Agency. Lucentis $10 \mathrm{mg} / \mathrm{ml}$ solution for injection. Summary of product characteristics. https://www.ema.europa.eu/en/documents/productinformation/lucentis-epar-product-information_en.pdf. Accessed 14 Aug 2021.

7. European Medicines Agency. Avastin $25 \mathrm{mg} / \mathrm{ml}$ concentrate for solution for infusion. Summary of product characteristcs. https://www.ema.europa.eu/en/ documents/product-information/avastin-epar-product-information_en.pdf. Accessed 29 Apr 2021.

8. Diabetic Retinopathy Clinical Research Network, Wells JA, Glassman AR, Ayala AR, Jampol LM, Aiello LP, et al. Aflibercept, bevacizumab, or ranibizumab for diabetic macular edema. N Engl J Med. 2015;372:1193-203.

9. CATT Research Group, Martin DF, Maguire MG, Ying GS, Grunwald JE, Fine SL, et al. Ranibizumab and bevacizumab for neovascular age-related macular degeneration. N Engl J Med. 2011;364:1897-908.

10. Li E, Donati S, Lindsley KB, Krzystolik MG, Virgili G. Treatment regimens for administration of anti-vascular endothelial growth factor agents for neovascular age-related macular degeneration. Cochrane Database Syst Rev. 2020;5: CD012208.

11. Mahmood S, Roberts SA, Aslam TM, Parkes J, Barugh K, Bishop PN, et al. Routine versus as-needed bevacizumab with 12-weekly assessment intervals for neovascular age-related macular degeneration: 92-week results of the GMAN trial. Ophthalmology. 2015;122:1348-55.

12. Berg K, Pedersen TR, Sandvik L, Bragadottir R. Comparison of ranibizumab and bevacizumab for neovascular age-related macular degeneration according to LUCAS treat-and-extend protocol. Ophthalmology. 2015;122:146-52.

13. Schmidt-Erfurth U, Kaiser PK, Korobelnik JF, Brown DM, Chong V, Nguyen QD, et al. Intravitreal aflibercept injection for neovascular age-related macular degeneration: ninety-six-week results of the VIEW studies. Ophthalmology. 2014;121:193-201.

14. Korobelnik JF, Holz FG, Roider J, Ogura Y, Simader C, Schmidt-Erfurth U, et al. Intravitreal aflibercept injection for macular edema resulting from central retinal vein occlusion: one-year results of the phase 3 GALILEO study. Ophthalmology. 2014;121:202-8.

15. Korobelnik JF, Do DV, Schmidt-Erfurth U, Boyer DS, Holz FG, Heier JS, et al. Intravitreal aflibercept for diabetic macular edema. Ophthalmology. 2014;121:2247-54.

16. Heier JS, Brown DM, Chong V, Korobelnik JF, Kaiser PK, Nguyen QD, et al. Intravitreal aflibercept (VEGF trap-eye) in wet age-related macular degeneration. Ophthalmology. 2012;119:2537-48.

17. Clark WL, Boyer DS, Heier JS, Brown DM, Haller JA, Vitti R, et al. Intravitreal aflibercept for macular edema following branch retinal vein occlusion: 52-week results of the VIBRANT study. Ophthalmology. 2016;123:330-6.

18. Brown DM, Heier JS, Clark WL, Boyer DS, Vitti R, Berliner AJ, et al. Intravitreal aflibercept injection for macular edema secondary to central retinal vein occlusion: 1-year results from the phase 3 COPERNICUS study. Am J Ophthalmol. 2013;155:429-37.e7

19. European Medicines Agency. Eylea $40 \mathrm{mg} / \mathrm{ml}$ solution for injection in pre-filled syringe. Summary of product characteristics. https://www.ema.europa.eu/en/ documents/product-information/eylea-epar-product-information_en.pdf. Accessed 14 Aug 2021.

20. Ohji M, Takahashi K, Okada AA, Kobayashi M, Matsuda Y, Terano Y, et al. Efficacy and safety of intravitreal aflibercept treat-and-extend regimens in exudative age-related macular degeneration: 52- and 96-week findings from ALTAIR: a randomized controlled trial. Adv Ther. 2020;37:1173-87.

21. Souied EH. Efficacy of intravitreal aflibercept treat-and-extend regimen over 2 years for neovascular age-related macular degeneration: ARIES study. Investig Ophthalmol Vis. Sci 2020;61:4260.

22. Barmas-Alamdari D, D'Souza HS, Kapoor KG, Wagner AL. Intravitreal Ziv-aflibercept: a comprehensive review. Semin Ophthalmol. 2019;34:420-35.

23. Dugel PU, Koh A, Ogura $Y$, Jaffe GJ, Schmidt-Erfurth U, Brown DM, et al. HAWK and HARRIER: phase 3, multicenter, randomized, double-masked trials of brolucizumab for neovascular age-related macular degeneration. Ophthalmology. 2020;127:72-84.

24. Baumal CR, Bodaghi B, Singer M, Tanzer DJ, Seres A, Joshi MR, et al. Expert opinion on management of intraocular inflammation, retinal vasculitis, and vascular occlusion after brolucizumab treatment. Ophthalmol Retina. 2021;5:519-27.

25. Baumal CR, Spaide RF, Vajzovic L, Freund KB, Walter SD, John V, et al. Retinal vasculitis and intraocular inflammation after intravitreal injection of brolucizumab. Ophthalmology. 2020;127:1345-59.

26. Bulirsch LM, Sassmannshausen M, Nadal J, Liegl R, Thiele S, Holz FG. Short-term real-world outcomes following intravitreal brolucizumab for neovascular AMD: SHIFT study. Br J Ophthalmol. 2021. https://doi.org/10.1136/bjophthalmol-2020318672.
27. Haug SJ, Hien DL, Uludag G, Ngoc TT, Lajevardi S, Halim MS, et al. Retinal arterial occlusive vasculitis following intravitreal brolucizumab administration. Am J Ophthalmol Case Rep. 2020;18:100680.

28. Jain A, Chea S, Matsumiya W, Halim MS, Yasar C, Kuang G, et al. Severe vision loss secondary to retinal arteriolar occlusions after multiple intravitreal brolucizumab administrations. Am J Ophthalmol Case Rep. 2020;18:100687.

29. Mones J, Srivastava SK, Jaffe GJ, Tadayoni R, Albini TA, Kaiser PK, et al. Risk of inflammation, retinal vasculitis, and retinal occlusion-related events with brolucizumab: post hoc review of HAWK and HARRIER. Ophthalmology. 2021;128:1050-9.

30. Novartis AG. Novartis reports one year results of Phase III MERLIN study evaluating Beovu ${ }^{\oplus}$ every four week dosing and provides update on Beovu clinical program. Basel 28 May 21. https://www.novartis.com/news/media-releases/ novartis-reports-one-year-results-phase-iii-merlin-study-evaluating-beovuevery-four-week-dosing-and-provides-update-beovu-clinical-program. Accessed 04 June 2021.

31. Liu K, Song Y, Xu G, Ye J, Wu Z, Liu X, et al. Conbercept for treatment of neovascular age-related macular degeneration: results of the randomized phase 3 PHOENIX study. Am J Ophthalmol. 2019;197:156-67.

32. Sharma A, Kumar N, Kuppermann BD, Bandello F. Abicipar pegol: the nonmonoclonal antibody anti-VEGF. Eye. 2020;34:797-801.

33. Kunimoto $D$, Yoon $\mathrm{YH}$, Wykoff CC, Chang A, Khurana RN, Maturi RK, et al. Efficacy and safety of abicipar in neovascular age-related macular degeneration: 52week results of phase 3 randomized controlled study. Ophthalmology. 2020;127:1331-44.

34. Kuppermann BD. Quarterly administration of the DARPin therapeutic abicipar in neovascular age-related macular degeneration. Investig Ophthalmol Vis Sci. 2020;61:5125.

35. Molecular Partners. Allergan and molecular partners announce topline safety results from MAPLE study of abicipar pegol. 02 April 2019. https://investors. molecularpartners.com/news-releases/news-release-details/allergan-andmolecular-partners-announce-topline-safety-results. Accessed 10 Aug 2019.

36. Heier JS, Singh RP, Wykoff CC, Csaky KG, Lai TYY, Loewenstein A, et al. The angiopoietin/TIE pathway in retinal vascular diseases: a review. Retina. 2021;41:1-19.

37. Khanani AM, Patel SS, Ferrone PJ, Osborne A, Sahni J, Grzeschik S, et al. Efficacy of every four monthly and quarterly dosing of faricimab vs ranibizumab in neovascular age-related macular degeneration: the STAIRWAY phase 2 randomized clinical trial. JAMA Ophthalmol. 2020;138:964-72.

38. Sahni J, Dugel PU, Patel SS, Chittum ME, Berger B, Del Valle Rubido M, et al. Safety and efficacy of different doses and regimens of faricimab vs ranibizumab in neovascular age-related macular degeneration: the AVENUE phase 2 randomized clinical trial. JAMA Ophthalmol. 2020;138:955-63.

39. Sahni J, Patel SS, Dugel PU, Khanani AM, Jhaveri CD, Wykoff $C C$, et al. Simultaneous inhibition of angiopoietin-2 and vascular endothelial growth factor-A with faricimab in diabetic macular edema: BOULEVARD phase 2 randomized trial. Ophthalmology. 2019;126:1155-70.

40. Danzig CJL H, Guibord P, Silverman D, Quezada Ruiz C, Stoilov I, Haskova Z. Clinical effects of blocking Ang-2 and VEGF with faricimab in the phase 2 STAIRWAY trial. Investig Ophthalmol Vis Sci. 2020;61:1160.

41. Hoffmann-La Roche. Roche's faricimab meets primary endpoint in two global phase III studies and shows potential to extend time between treatments up to 16 weeks for people with neovascular age-related macular degeneration. Basel 2501 2021. https://www.roche.com/media/releases/med-cor-2021-01-25.htm. Accessed 14 Aug 2021.

42. Beckmann R, Jensen K, Fenn S, Speck J, Krause K, Meier A, et al. DutaFabs are engineered therapeutic Fab fragments that can bind two targets simultaneously. Nat Commun. 2021;12:708.

43. Li F, Mahato RI. Bioconjugate therapeutics: current progress and future perspective. Mol Pharm. 2017;14:1321-4.

44. Al-Khersan H, Hussain RM, Ciulla TA, Dugel PU. Innovative therapies for neovascular age-related macular degeneration. Expert Opin Pharmacother. 2019;20:1879-91.

45. Patel SS, Janer D, Miller B, Ehrlich JS, Perlroth V, Velazquez-Martin JP. Updated results of phase $1 \mathrm{~b}$ study of KSI-301, an anti-VEGF antibody biopolymer conjugate with extended durability, in WAMD, DME, and RVO. Investig Ophthalmol Vis Sci. 2020;61:4286

46. Kodiak Sciences Inc. Kodiak sciences announces 1-year durability, efficacy and safety data from ongoing phase $1 \mathrm{~b}$ study of KSI-301 in patients with wet agerelated macular degeneration, diabetic macular edema and retinal vein occlusion at the angiogenesis, exudation and degeneration 2021 annual meeting. 13.02.2021. https://www.prnewswire.com/news-releases/kodiaksciences-announces-1-year-durability-efficacy-and-safety-data-from-ongoingphase-1 b-study-of-ksi-301-in-patients-with-wet-age-related-maculardegeneration-diabetic-macular-edema-and-retinal-vein-occlusion-at-theangiogene-301227910.html. Accessed 4 June 2021. 
47. Tsujinaka H, Fu J, Shen J, Yu Y, Hafiz Z, Kays J, et al. Sustained treatment of retinal vascular diseases with self-aggregating sunitinib microparticles. Nat Commun. 2020;11:694

48. Graybug Vision. Graybug vision presents top line results of phase 1/2a ADAGIO study at hawaiian eye \& retina 2019. https://www.graybug.vision/graybugvision-presents-top-line-results-of-phase-1-2a-adagio-study-at-hawaiian-eyeretina-2019/. Accessed 29 Apr 2021.

49. Avery RLW, Chang JA, Guymer R, Wickremashinghe S, Bell N, Vantipalli S, et al. Preliminary findings from a phase 1 trial evaluating the safety, tolerability and biological activity of OTX-TKI, a hydrogel-based, sustained release intravitreal axitinib implant, in subjects with neovascular age-related macular degeneration. Retina Society Annual Scientific Meeting, Virtual. 2020. Allegro Ophthalmics LLC. Allegro ophthalmics announces positive topline results from DEL MAR phase $2 b$ stage 2 trial evaluating luminate ${ }^{\circledast}$ in patients with diabetic macular edema. https://www.retinasociety.org/content/ meetingarchive/2020/avery-robert-preliminary-findings-from-a-phase-1.pdf. Accessed 10 Nov 21.

50. Dunn EN, Hariprasad SM, Sheth VS. An overview of the fovista and rinucumab trials and the fate of anti-PDGF medications. Ophthalmic Surg Lasers Imaging Retina. 2017;48:100-4.

51. Apellis Pharma. Apellis provides 24-month update from phase $1 \mathrm{~b}$ study of pegcetacoplan in patients with geographic atrophy. 13.04.2021. https:// investors.apellis.com/news-releases/news-release-details/apellis-provides-24month-update-phase-1b-study-pegcetacoplan. Accessed 22 June 2021.

52. Quiroz-Mercado H, Boyer DS, Campochiaro PA, Heier JS, Kaiser PK, Kornfield J, et al. Randomized, prospective, double-masked, controlled phase $2 \mathrm{~b}$ trial to evaluate the safety \& efficacy of ALG-1001 (Luminate ${ }^{\circledast}$ ) in diabetic macular edema. Investig Ophthalmol Vis Sci. 2018;59:1960.

53. Allegro Ophthalmics LLC. Allegro ophthalmics announces positive topline results from DEL MAR phase $2 \mathrm{~b}$ stage 2 trial evaluating luminate ${ }^{\circledR}$ in patients with diabetic macular edema. 13.10.2016. https://www.allegroeye.com/allegroophthalmics-announces-positive-topline-results-from-del-mar-phase-2b-trialevaluating-luminate-in-patients-with-diabetic-macular-edema/. Accessed $28 \mathrm{Apr}$ 2021.

54. European Medicines Agency. Rhokiinsa 200 micrograms/ml eye drops, solution Summary of product characteristics https://www.ema.europa.eu/en/documents/ product-information/rhokiinsa-epar-product-information_en.pdf Accessed 7 Dec 2021.

55. Hoy SM. Netarsudil ophthalmic solution 0.02\%: first global approval. Drugs. 2018;78:389-96.

56. Ahmadieh H, Nourinia R, Hafezi-Moghadam A, Sabbaghi H, Nakao S, Zandi S, et al. Intravitreal injection of a Rho-kinase inhibitor (fasudil) combined with bevacizumab versus bevacizumab monotherapy for diabetic macular oedema: a pilot randomised clinical trial. Br J Ophthalmol. 2019;103:922-7.

57. Jacobo SM, Deangelis MM, Kim IK, Kazlauskas A. Age-related macular degeneration-associated silent polymorphisms in HtrA1 impair its ability to antagonize insulin-like growth factor 1. Mol Cell Biol. 2013;33:1976-90.

58. Tom I, Pham VC, Katschke KJ Jr, Li W, Liang WC, Gutierrez J, et al. Development of a therapeutic anti-HtrA1 antibody and the identification of DKK3 as a pharmacodynamic biomarker in geographic atrophy. Proc Natl Acad Sci USA 2020;117:9952-63.

59. Khanani AM, Callanan D, Dreyer R, Chen S, Howard JG, Hopkins JJ, et al. End-ofstudy results for the ladder phase 2 trial of the port delivery system with ranibizumab for neovascular age-related macular degeneration. Ophthalmol Retina. 2021;5:775-87.

60. Hoffmann-La Roche. Phase III data show Roche's port delivery system with ranibizumab enabled over $98 \%$ of patients to go six months between treatments for neovascular age-related macular degeneration. 22.07.2020. https:// www.roche.com/media/releases/med-cor-2020-07-22b.htm. Accessed 14 Aug 2021.

61. Recchia F, Blotner S, Singh N, Barteselli G, Gune S. Ladder phase 2 trial of the port delivery system with ranibizumab (PDS) for neovascular AMD: end of study results. Investig Ophthalmol Vis Sci. 2020;61:1155.

62. Schmidt-Erfurth U, Garcia-Arumi J, Bandello F, Berg K, Chakravarthy U, Gerendas $B S$, et al. Guidelines for the management of diabetic macular edema by the European Society of Retina Specialists (EURETINA). Ophthalmologica. 2017;237:185-222

63. Garcia Layana A, Adan A, Ascaso FJ, Cabrera F, Donate J, Escobar Barranco J J et al. Use of intravitreal dexamethasone implants in the treatment of diabetic macular edema: expert recommendations using a Delphi approach. Eur J Ophthalmol. 2020;30:1042-52.

64. Mantopoulos D, Zhang R, Roth DB, Optimizing DME. Treatment with decreased injection frequency using long-term steroid implants. Ophthalmic Surg Lasers Imaging Retina. 2020;51:S22-9.
65. Rehak M, Busch C, Unterlauft JD, Jochmann C, Wiedemann P. Outcomes in diabetic macular edema switched directly or after a dexamethasone implant to a fluocinolone acetonide intravitreal implant following anti-VEGF treatment Acta Diabetol. 2020;57:469-78.

66. European Medicines Agency. OZURDEX 700 micrograms intravitreal implant in applicator. Summary of Product Characteristics. https://www.ema.europa.eu/en/ documents/product-information/ozurdex-epar-product-information_en.pdf. Accessed 14 Aug 2021.

67. Lightman S, Belfort R Jr., Naik RK, Lowder C, Foster CS, Rentz AM, et al. Visionrelated functioning outcomes of dexamethasone intravitreal implant in noninfectious intermediate or posterior uveitis. Investig Ophthalmol Vis Sci. 2013;54:4864-70.

68. Kuppermann BD, Haller JA, Bandello F, Loewenstein A, Jiao J, Li XY, et al. Onset and duration of visual acuity improvement after dexamethasone intravitreal implant in eyes with macular edema due to retinal vein occlusion. Retina. 2014;34:1743-9.

69. Boyer DS, Yoon YH, Belfort R Jr, Bandello F, Maturi RK, Augustin AJ, et al. Threeyear, randomized, sham-controlled trial of dexamethasone intravitreal implant in patients with diabetic macular edema. Ophthalmology. 2014;121:1904-14.

70. European Medicines Agency. ILUVIEN 190 micrograms intravitreal implant in applicator. Summary of product characteristics. https://www.medicines.org.uk/ emc/product/3061/smpc\#gref. Accessed 14 Aug 2021.

71. Pavesio C, Zierhut M, Bairi K, Comstock TL, Usner DW, Fluocinolone Acetonide Study G. Evaluation of an intravitreal fluocinolone acetonide implant versus standard systemic therapy in noninfectious posterior uveitis. Ophthalmology. 2010;117:567-75.e1.

72. Jaffe GJ, Pavesio CE, Study I. Effect of a fluocinolone acetonide insert on recurrence rates in noninfectious intermediate, posterior, or panuveitis: threeyear results. Ophthalmology. 2020;127:1395-404.

73. Jaffe GJ, Foster CS, Pavesio CE, Paggiarino DA, Riedel GE. Effect of an Injectable fluocinolone acetonide insert on recurrence rates in chronic noninfectious uveitis affecting the posterior segment: twelve-month results. Ophthalmology. 2019;126:601-10.

74. Yang Y, Bailey C, Holz FG, Eter N, Weber M, Baker C, et al. Long-term outcomes of phakic patients with diabetic macular oedema treated with intravitreal fluocinolone acetonide (FAc) implants. Eye. 2015;29:1173-80.

75. Campochiaro PA, Brown DM, Pearson A, Ciulla T, Boyer D, Holz FG, et al. Longterm benefit of sustained-delivery fluocinolone acetonide vitreous inserts for diabetic macular edema. Ophthalmology. 2011;118:626-35.e2.

76. Patel SR, Lin AS, Edelhauser HF, Prausnitz MR. Suprachoroidal drug delivery to the back of the eye using hollow microneedles. Pharm Res. 2011;28:166-76.

77. Patel SR, Berezovsky DE, McCarey BE, Zarnitsyn V, Edelhauser HF, Prausnitz MR. Targeted administration into the suprachoroidal space using a microneedle for drug delivery to the posterior segment of the eye. Investig Ophthalmol Vis Sci. 2012;53:4433-41.

78. Clearside Biomedical. Clearside biomedical announces SAPPHIRE phase 3 study of combination therapy in retinal vein occlusion did not meet its primary endpoint. https://ir.clearsidebio.com/news-releases/news-release-details/clearsidebiomedical-announces-sapphire-phase-3-study. 2021. Accessed 12 Aug 2021.

79. Yeh S, Khurana RN, Shah M, Henry CR, Wang RC, Kissner JM, et al. Efficacy and safety of suprachoroidal CLS-TA for macular edema secondary to noninfectious uveitis: phase 3 randomized trial. Ophthalmology. 2020;127:948-55.

80. European Medicines Agency. MabThera $100 \mathrm{mg}$ concentrate for solution for infusion. Summary of product characteristics. https://www.ema.europa.eu/en/ documents/product-information/mabthera-epar-product-information_en.pdf. Accessed 26 May 2021.

81. Kurz PA, Suhler EB, Choi D, Rosenbaum JT. Rituximab for treatment of ocular inflammatory disease: a series of four cases. Br J Ophthalmol. 2009;93:546-8.

82. Pelegrin L, Jakob E, Schmidt-Bacher A, Schwenger V, Becker M, Max R, et al. Experiences with rituximab for the treatment of autoimmune diseases with ocular involvement. J Rheumatol. 2014;41:84-90.

83. Suhler EB, Lim LL, Beardsley RM, Giles TR, Pasadhika S, Lee ST, et al. Rituximab therapy for refractory scleritis: results of a phase I/II dose-ranging, randomized, clinical trial. Ophthalmology. 2014;121:1885-91.

84. Suhler EB, Lim LL, Beardsley RM, Giles TR, Pasadhika S, Lee ST, et al. Rituximab therapy for refractory orbital inflammation: results of a phase $1 / 2$, dose-ranging, randomized clinical trial. JAMA Ophthalmol. 2014;132:572-8.

85. Davatchi F, Shams H, Rezaipoor M, Sadeghi-Abdollahi B, Shahram F, Nadji A, et al. Rituximab in intractable ocular lesions of Behcet's disease; randomized single-blind control study (pilot study). Int J Rheum Dis. 2010;13:246-52.

86. Messina MJ, Rodegher M, Scotti R, Martinelli V. Treatment of myelitis in Behcet's disease with rituximab. BMJ Case Rep. 2014;2014:bcr2014204366.

87. Sadreddini $\mathrm{S}$, Noshad $\mathrm{H}$, Molaeefard M, Noshad R. Treatment of retinal vasculitis in Behcet's disease with rituximab. Mod Rheumatol. 2008;18:306-8. 
88. Zhao BH, Oswald AE. Improved clinical control of a challenging case of Behcet's disease with rituximab therapy. Clin Rheumatol. 2014;33:149-50.

89. European Medicines Agency. LEMTRADA $12 \mathrm{mg}$ concentrate for solution for infusion. Summary of product characteristics. https://www.ema.europa.eu/en/ documents/product-information/lemtrada-epar-product-information_en.pdf. Accessed 28 May 2021.

90. Willis MD, Pickersgill TP, Robertson NP, Lee RWJ, Dick AD, Carreño E. Alemtuzumab-induced remission of multiple sclerosis-associated uveitis. Int Ophthalmol. 2017;37:1229-33.

91. Daniels GH, Vladic A, Brinar V, Zavalishin I, Valente W, Oyuela P, et al. Alemtuzumab-related thyroid dysfunction in a phase 2 trial of patients with relapsing-remitting multiple sclerosis. J Clin Endocrinol Metab. 2014;99:80-9.

92. Azevedo CJ, Kutz C, Dix A, Boster A, Sanossian N, Kaplan J. Intracerebral haemorrhage during alemtuzumab administration. Lancet Neurol. 2019;18:329-31.

93. European Medicines Agency. Meeting highlights from the Pharmacovigilance Risk Assessment Committee (PRAC). 2019. https://www.ema.europa.eu/en/ documents/agenda/agenda-prac-draft-agenda-meeting-8-11-april-2019_en.pdf. Accessed 7 May 2021.

94. Heier JSCPHA. Results of cohorts 1-5 for the RGX-314 phase I/lla study of gene therapy for neovascular wAMD. Retina Subspecialty Day, San Francisco, California: American Academy of Ophthalmology; 2019.

95. Regenxbio Inc. RGX-314 Gene therapy administered in the suprachoroidal space for participants with neovascular age-related macular degeneration (nAMD). Rockville, Md. http://ir.regenxbio.com/news-releases/news-release-details/ regenxbio-presents-positive-initial-data-phase-ii-altitudetm (Accessed $7 \mathrm{Dec}$ 2021).

96. Busbee BB, Khanani DS, Wykoff AM, Pieramici CC, Regillo DJ, Danzig C, et al. 1 Study of Intravitreal Gene Therapy with ADVM-022 for neovascular AMD (OPTIC Trial). Investig Ophthalmol Vis Sci. 2021;62:1154.

97. Gyroscope Therapeutics Limited. HORIZON: a phase II study to evaluate the safety and efficacy of two doses of GT005. 28.09.2020 https://ClinicalTrials.gov/ show/NCT04566445. Accessed 14 Aug 2021.

98. Gyroscope Therapeutics Limited. Gyroscope therapeutics announces positive interim data from phase I/II FOCUS trial of investigational gene therapy GT005. 2021. https://www.businesswire.com/news/home/20210212005080/en/GyroscopeTherapeutics-Announces-Positive-Interim-Data-from-Phase-III-FOCUS-Trial-ofInvestigational-Gene-Therapy-GT005. Accessed 14 Aug 2021.

99. Gong D, Kras A, Miller JB. Application of deep learning for diagnosing, classifying, and treating age-related macular degeneration. Semin Ophthalmol. 2021;36:198-204.

100. Craven ER, Walters T, Christie WC, Day DG, Lewis RA, Goodkin ML, et al. 24Month phase I/II clinical trial of bimatoprost sustained-release implant (Bimatoprost SR) in glaucoma patients. Drugs. 2020;80:167-79.

101. Lewis RA, Christie WC, Day DG, Craven ER, Walters T, Bejanian M, et al. Bimatoprost sustained-release implants for glaucoma therapy: 6-month results from a phase I/II clinical trial. Am J Ophthalmol. 2017;175:137-47.

102. Medeiros FA, Walters TR, Kolko M, Coote M, Bejanian M, Goodkin ML, et al. Phase 3, randomized, 20-month study of bimatoprost implant in open-angle glaucoma and ocular hypertension (ARTEMIS 1). Ophthalmology. 2020;127:1627-41.

103. Whitcup SM, Robinson MR. Development of a dexamethasone intravitreal implant for the treatment of noninfectious posterior segment uveitis. Ann NY Acad Sci. 2015;1358:1-12.

104. Mansberger SL, Conley J, Verhoeven RS, Blackwell K, Depenbusch M, Knox T, et al. Interim analysis of low dose ENV515 travoprost XR with 11 month duration followed by dose escalation and 28 day efficacy evaluation of high dose ENV515. Investig Ophthalmol Vis Sci. 2017;58:2110.

105. Goldstein MH, Goldberg D, Walters TR, Vantipalli S, Braun E, Metzinger JL. Evaluating safety, tolerability and efficacy of an intracameral hydrogel-based travoprost implant in subjects with glaucoma-phase 1 Trial. Investig Ophthalmol Vis Sci. 2020;61:4266.

106. Vantipalli S, Sall KN, Stein E, Schenker H, Mulaney J, Smyth-Medina R, et al. Evaluation of the Safety and Efficacy of OTX-TP, an intracanalicular travoprost insert, for the treatment of patients with open-angle glaucoma or ocular hypertension: a phase 3 study. Investig Ophthalmol Vis Sci. 2020;61:3488.

107. Kesav NP, Young CEC, Ertel MK, Seibold LK, Kahook MY. Sustained-release drug delivery systems for the treatment of glaucoma. Int J Ophthalmol. 2021;14:148-59.

108. Brandt JD, DuBiner HB, Benza R, Sall KN, Walker GA, Semba CP. Long-term safety and efficacy of a sustained-release bimatoprost ocular ring. Ophthalmology. 2017;124:1565-6.

109. Wong TT, Novack GD, Natarajan JV, Ho CL, Htoon HM, Venkatraman SS. Nanomedicine for glaucoma: sustained release latanoprost offers a new therapeutic option with substantial benefits over eyedrops. Drug Deliv Transl Res. 2014;4:303-9.
110. Xu J, Ge Y, Bu R, Zhang A, Feng S, Wang J, et al. Co-delivery of latanoprost and timolol from micelles-laden contact lenses for the treatment of glaucoma. J Control Release. 2019;305:18-28.

111. Gazzard G, Konstantakopoulou E, Garway-Heath D, Garg A, Vickerstaff V, Hunter $\mathrm{R}$, et al. Selective laser trabeculoplasty versus eye drops for first-line treatment of ocular hypertension and glaucoma (LiGHT): a multicentre randomised controlled trial. Lancet. 2019;393:1505-16.

112. Khawaja AP, Campbell JH, Kirby N, Chandwani HS, Keyzor I, Parekh M, et al. Realworld outcomes of selective laser trabeculoplasty in the United Kingdom. Ophthalmology. 2020;127:748-57.

113. Pillunat KR, Spoerl E, Elfes G, Pillunat LE. Preoperative intraocular pressure as a predictor of selective laser trabeculoplasty efficacy. Acta Ophthalmol. 2016;94:692-6

114. Hodge WG, Damji KF, Rock W, Buhrmann R, Bovell AM, Pan Y, et al. predicts selective laser trabeculoplasty success at 1 year post-treatment: results from a randomised clinical trial. Br J Ophthalmol. 2005;89:1157-60.

115. Groth SL, Albeiruti $E$, Nunez M, Fajardo R, Sharpsten L, Loewen N, et al. SALT trial: steroids after laser trabeculoplasty: impact of short-term anti-inflammatory treatment on selective laser trabeculoplasty efficacy. Ophthalmology. 2019;126:1511-6.

\section{ACKNOWLEDGEMENTS}

This document, and its development, were independent of the funder. None of the authors have received any funding for contributing to the development of this paper. The Group retained final control of all the content and editorial decisions. Editorial assistance for this review was supported by Allergan via an independent and unrestricted research grant. Allergan had the opportunity to review the final version of the manuscript to address any factual inaccuracies or request the redaction of information deemed to be proprietary or confidential and ensure that study support was disclosed.

\section{FUNDING}

Editorial assistance was provided to the authors by Dr Mark Hillen, BSc, Ph.D., through an unrestricted grant, funded by Allergan International plc, Dublin, Ireland. Publication costs were also provided through an unrestricted education grant from Allergan International at the request of the lead author. All authors met the ICMJE authorship criteria. Neither honoraria nor payments were made for authorship and authors retained full control over the paper.

\section{COMPETING INTERESTS}

FG: Institutional research grants from: Allergan, Bayer, Boehringer Ingelheim, Chengdu Pharma, Clearside, Novartis, Roche. Travel Grants from Allergan, Bayer, Novartis. Lecture fees from: Allergan, Alimera, Heidelberg, Bayer, Novartis, Roche, Ad board/Consultancy for Allergan, Alimera, Apellis, Bayer, Boehringer Ingelheim, Novartis, Roche. Editorial board member for Eye at time of publication. RG: Consultancy for Novartis, Bayer, Roche, Alimera/Allergan, Santen, Research/Educational grants from Bayer, Novartis. CR: Travel grants from Novartis, Bayer, Allergan; Advisory board/consultancy for Allergan, Alimera, Novartis. RB: Consultancy for Santen, Allergan, Thea. Editorial board member for Eye at time of publication. SS: Research grants from Novartis, Bayer, Allergan, Roche, Boehringer Ingelheim, and Optos Plc, travel grants from Novartis and Bayer, speaker fees from Novartis, Bayer, and Optos Plc, and attending advisory board meetings for Novartis, Bayer, Allergan, Roche, Boehringer Ingelheim, Optos Plc, Oxurion, Opthea, Apellis, Oculis and Heidelberg Engineering. Editor in Chief of Eye at time of publication. SD: remunerated consultancies for Allergan, Janssen, Boehringer Ingelheim, and Circadian Therapeutics; travel grants/honoraria from Ely Lilly and Novartis, Bayer and is/has been a PI on pharma research clinical trials sponsored by Novartis, Bayer, Allergan, Roche. IT: None.

\section{ADDITIONAL INFORMATION}

Correspondence and requests for materials should be addressed to Faruque Ghanchi.

Reprints and permission information is available at http://www.nature.com/ reprints

Publisher's note Springer Nature remains neutral with regard to jurisdictional claims in published maps and institutional affiliations. 
Open Access This article is licensed under a Creative Commons At c) Atribution 4.0 International License, which permits use, sharing,
adaptation, distribution and reproduction in any medium or format, as long as you give appropriate credit to the original author(s) and the source, provide a link to the Creative Commons license, and indicate if changes were made. The images or other third party material in this article are included in the article's Creative Commons license, unless indicated otherwise in a credit line to the material. If material is not included in the article's Creative Commons license and your intended use is not permitted by statutory regulation or exceeds the permitted use, you will need to obtain permission directly from the copyright holder. To view a copy of this license, visit http://creativecommons. org/licenses/by/4.0/.

(c) The Author(s) 2021 